\title{
Systemic Host Modulation Therapy in the Treatment of Periodontal Disease
}

\author{
Muna S Elburki* \\ Department of Periodontics, Faculty of Dentistry, University of Benghazi, Libya
}

Received: 制 June 06, 2018; Published: 制August 01, 2018

*Corresponding author: Muna S Elburki, Department of Periodontics, Faculty of Dentistry, University of Benghazi, Benghazi, Libya

\begin{abstract}
Abbreviations: SBU: Stony Brook University; HSDM: Harvard School of Dental Medicine; MMPs: Matrix Metalloproteinases; NSAIDs: Non-Steroidal Anti-Inflammatory Drugs; SDD: Sub-Antimicrobial-Doses of Doxycycline; CMTs: Chemically Modified Tetracyclines; ARDS: Acute Respiratory Distress Syndrome; CMC: Chemically Modified Curcumin; NOS: Nitric Oxide synthase
\end{abstract}

\section{Mini Review}

In the mid1980s, Williams and his colleagues at the Harvard School of Dental Medicine (HSDM), and Golub and his group at Stony Brook University (SBU), addressed the importance of the Host Modulation Therapy (HMT) in periodontal disease by demonstrating

a) that non-steroidal anti-inflammatory drugs or NSAIDs such as flurbiprofen can inhibit alveolar bone loss in animal studies and in human clinical trials [1-4] and

b) that Tetracyclines, by NON-antibacterial mechanisms can inhibit host-derived Matrix metalloproteinases (MMPs) and osteoclast activity to suppress collagenase and bone resorption, processes essential for periodontal and other (e.g., Rheumatoid arthritis) diseases [5-7].

This paradigm shift at that time, with a focus on the host response, led to the search for novel therapies for periodontitis. These host-modulating therapies included:

\section{The Non-Steroidal Anti-Inflammatory Drugs (NSAIDs)}

These are commonly used to treat pain and inflammation and include the inhibitors of the prostaglandins and other arachidonic acid metabolites which are associated with the pathogenesis of periodontitis $[3,8,9]$. Therefore, it was logical to use inhibitors of arachidonic acid metabolites, such as the NSAIDs, in treatment of periodontitis [1-4]. However, NSAIDs are frequently associated with gastrointestinal side effects, such as bleeding or perforation of gastroduodenal ulcers [10] and increased risk of CVD [11] and cannot be recommended for long-term use as a host modulating therapy [12]. Also, flurbiprofen, systemically administered did produce a "rebound" effect i.e., after stopping NSAID treatment, the disease severity rebounded and become even worse than that before this treatment was initiated [13].

\section{The Tetracyclines}

These are broad-spectrum antibiotics that unexpectedly were found to inhibit host-derived MMPs [14-16]. This property was first identified by Golub et al. [5-7] who demonstrated that a semi-synthetic tetracycline, minocycline had the ability to reduce pathologically-excessive collagenase activity even in a germ-free diabetic rat model. This was a seminal discovery for two reasons:

a) Because it identified for the first time that tetracyclines can inhibit mammalian (host-derived) collagenases and other MMPs

b) By mechanisms that were unrelated to their well-known antimicrobial activity [7].

Additional studies soon verified and expanded the use of tetracyclines as a potential treatment for periodontal disease because of these newly-recognized characteristics and their ability to inhibit pathologically-elevated collagenase activity $[14,15,17,18]$. The success of the tetracyclines was propelled into mass-market application when several novel formulations of TCs were developed including:

a) NON or sub-antimicrobial-doses of doxycycline (SDD), which were ultimately FDA approved for the treatment of periodontal disease (Periostat @) [14,15,19-21] and later a NOVEL sustained-release formulation of SDD which was approved for treating the chronic inflammatory skin condition, rosacea (Oracea ${ }^{\circledR}$ ).

Although a number of researchers have tried to develop MMPinhibitors (MMP-Is) to inhibit connective tissue degradation associated with inflammatory and other diseases, their efforts were not successful [22-24] for review); the only US-FDA approved systemically administered drug as MMP-Is are those based on TCs. 
Doxycycline, when administered as a regular dose tetracycline, like most, if not all antibiotics, produces complications such as gastrointestinal disturbance, increased photosensitivity, and the emergence of antibiotic-resistant microorganisms [20,21]. They found, however, that lowering the dose of doxycycline to produce peak ( $\mathrm{C}$ max) blood levels, $<1 \mu \mathrm{g} / \mathrm{mL}$, could eliminate these complications while providing essentially the same MMP inhibitory properties see $[14,15,17]$ Other benefits to SDD include its low IC50 value and its proven safety in clinical trials on patients with both dental and medical conditions [16,25].

These data appear to be consistent with an earlier study by Lee et al. [ 5,7,10] in which humans requiring extensive periodontal surgery, allowing analysis of the excised gingival tissues, demonstrated that a combination of a low dose NSAID (which by itself was ineffective) combined with SDD synergistically reduced MMPs (collagenase, gelatinase and even PMNL elastase) in the excised gingival tissues. With SDD as an effective treatment for periodontal disease Golub et al. [ 5,6,7] then synthesized non-antibacterial analogs of tetracyclines and the first of these chemically-modified tetracyclines (4-dedimethylamino tetracycline; CMT-1) was generated by his team in 1987 [26] by the removal of the dimethyl amino group from carbon- 4 of the TC molecule which is responsible for the antibiotic activity of this class of compounds. This chemical modification did not impair the MMP-I activity and some of these CMTs (such as CMT-3; 6-demethyl 6 deoxy 4-dedimethyl amino tetracycline) were in fact more potent MMP-Is than the antibiotic TCs [26]. A discussion of these follows.

\section{Tetracycline Derived MMP-Is}

Tetracycline analogs have been developed, i.e.., the chemicallymodified tetracyclines (CMTs), which have lost their antibiotic activity, but which retained their calcium and zinc binding sites at carbon-11 and carbon-12 (a $\beta$-diketone moiety) and their proteinases-inhibitory properties. These have shown efficacy in experimental periodontitis and other diseases in animal studies $[27,28]$ and in human clinical trials (see below). CMTs became an attractive option because, in theory, they would be able to reduce these pathologically elevated collagenase levels at higher doses than sub-antimicrobial-dose doxycycline without creating drugresistant bacteria. A series of CMTs were synthesized and were tested for their potency as inhibitors of MMP activity [14]. Several CMTs such as CMT-1, CMT-2, CMT-3, and CMT- 6 showed potential for the reduction of collagenase activity in various diseases. The lead compound, CMT-3, has shown efficacy in a pig-model of acute respiratory distress syndrome (ARDS), diabetes, arthritis and cancer [15,16,25-29] and in phase II clinical trials in humans with Kaposi's sarcoma, as an anti-angiogenesis drug. However, this compound did result in adverse events, specifically increased photosensitivity in these subjects [30-35] and to date, has not been developed further. However, a pilot study by Ryan et al. [16] using a much lower oral/systemic dose of CMT-3 (i.e., 10mg/day), rather than the $50-150 \mathrm{mg} /$ day in the phase I and II studies on cancer patients [33-35] did appear to reduce IL-1 $\beta$ and MMP-8 in human periodontal pockets [36].

\section{Bisphosphonates}

Are widely used in the treatment of systemic metabolic bone diseases as a result of their ability to inhibit osteoclastmediated bone resorption. Because of their ability to increase the differentiation of fibroblast into osteoblast and to inhibit the activity of osteoclasts, it was tested in clinical trials in subjects with periodontitis $[37,38]$. However, within the last few years, the literature has indicated that bisphosphonate use, particularly intravenous preparations, may be linked to osteonecrosis of the jaws, and that their efficacy in periodontitis has not been consistent $[39,40]$.

\section{Lipid Derived Mediators}

Recently, new families of lipid-derived mediators, such as lipoxins, protectins, and resolvins, were found to possess potent anti-inflammatory/proresolving activity in vivo, specifically their ability to resolve acute inflammation [41]. More recently Naqvi et al. (2014) demonstrated that docosahexaenoic supplementation (an omega- 6 fatty acid) combined with low dose aspirin significantly reduced periodontitis and gingival inflammation in humans in a double-blind placebo-controlled trial of 3 months duration [39].

\section{Chemically Modified Curcumins (CMCs)}

Numerous investigators have described various pharmacologic strategies to modulate the host response during periodontal disease, however only a non-antimicrobial tetracycline formulation is FDA approved for these patients. However, a significant drawback of the approved SDD is that this novel low-dose formulation cannot be increased in order to prevent the emergence of antibiotic-resistant bacteria. Evidence for the role of MMPs, cytokines, and other mediators in the pathogenesis of periodontal disease differentiates them as possible targets for a host modulation therapies. The properties of TCs and CMTs as inhibitors of MMPs is associated, at least in part, with zinc-binding by the compounds, in the catalytic domain of these proteinases [42]. Curcumin, which also has this enolic beta-diketone moiety was chosen as the next generation compound for MMP inhibition. However, due to curcumin's low absorption into the body and high rate of metabolism [43] it was modified in an attempt to increase its bioavailability and efficacy [42]. In order to increase the solubility and zinc-binding of curcumin, a series of curcumin analogs were synthesized with a carbonyl substituent at the C-4 position. Golub and his group recently designed new MMP-inhibitor compounds containing the same zinc-binding site as the tetracyclines, but which are bi-cyclic phenolic compounds rather than teracycles.

Golub and his group demonstrate that systemic administration of a newly developed chemically modified curcumin (CMC2.24) in locally and systemically-induced model of experimental periodontitis significantly inhibited alveolar bone loss and attenuated the severity of local and systemic inflammation [4446]. Moreover, this novel chemically-modified-curcumin (CMC2.24) appears to reduce the pathologically-excessive levels of inducible MMPs to near normal levels but appears to have no significant effect on the constitutive MMPs required for physiologic connective tissue turnover. Additionally, a study performed on human monocytes 
stimulated with endotoxin illustrated that CMC 2.24 was capable of reducing excessive levels of inflammatory mediators such as IL$1 \beta$, TNF- $\alpha$, PGE2, and MCP-1 [42]. The same monocyte study also indicated that CMC 2.24 reduced the elevated levels of MMP-2, -9, -8 , and -13 down to normal levels with potential host-modulation action [42]. There are additional novel host response therapeutic approaches (i.e. Nitric Oxide synthase (NOS) inhibitors, p38 MAPK inhibitors, NF- $\kappa \mathrm{B}$ family inhibitors and TNF antagonists) to treat periodontal diseases currently in preclinical studies in various animal models with potential host-modulation action [47].

\section{References}

1. Howell T Howard, Ray C Williams (1993) Nonsteroidal antiinflammatory drugs as inhibitors of periodontal disease progression. Critical Reviews in Oral Biology \& Medicine 4(2): 177-196.

2. Williams RC, Jeffcoat MK, Wechter WJ, Johnson HG, Kaplan ML, et al. (1984) Non-steroidal anti-inflammatory drug treatment of periodontitis in beagles. Journal of periodontal research 19(6): 633-637.

3. Williams RC, Jeffcoat MK, Howell TH, Hall CM, Johnson HG, et al. (1987) Indomethacin or flurbiprofen treatment of periodontitis in beagles: comparison of effect on bone loss. Journal of periodontal research 22(5): 403-407.

4. Williams RC, Jeffcoat MK, Howell TH, MS Reddy, Johnson HG, et al. (1988) Topical flurbiprofen treatment of periodontitis in beagles. Journal of periodontal research 23(3): 166-169.

5. Golub LM, Wolff M, Roberts S, Lee HM, Leung M, Payonk G (1994) Treating periodontal diseases by blocking tissue-destructive enzymes. JADA 125(2): 163-169.

6. Golub L, Ryan M, Williams R (1998) Modulation of the host response in the treatment of periodontitis. Dentistry Today 17(10): 102-106.

7. Golub LM, Lee HM, Lehrer G, Nemiroff A, Mc Namara TF, et al. (1983) Minocycline reduces gingival collagenolytic activity during diabetes. Preliminary observations and a proposed new mechanism of action. Journal of periodontal research 18(5): 516-526.

8. Klein D, Raisz L (1970) Prostaglandins: stimulation of bone resorption in tissue culture. Endocrinology 86(6): 1436-1440.

9. Goldhaber PL, Rabadjija, Beyer WR, Kornhauser A (1973) Bone resorption in tissue culture and its relevance to periodontal disease. ] Am Dent Assoc 87(5): 1027-1033.

10. Buttgereit Frank, Gerd R Burmester, Lee S Simon (2001) Gastrointestinal toxic side effects of nonsteroidal anti-inflammatory drugs and cyclooxygenase-2-specific inhibitors. The American journal of medicine 110(3): 13-19.

11. Graham David J, David Campen, Rita Hui, Michele Spence, Craig Cheetham, et al. (2005) Risk of acute myocardial infarction and sudden cardiac death in patients treated with cyclo-oxygenase 2 selective and non-selective non-steroidal anti-inflammatory drugs: nested casecontrol study. The Lancet 365(9458): 475-481.

12. Williams Ray C, Marjorie K Jeffcoat, T Howard Howell, Arturo Rolla, Derek Stubbs, et al. (1989) Altering the progression of human alveolar bone loss with the non-steroidal anti-inflammatory drug flurbiprofen. Journal of periodontology 60(9): 485-490.

13. Lichtenberger LM, Wang ZM, Romero JJ, Ulloa C, Perez JC, Giraud MN, et al. (1995) Non-steroidal anti-inflammatory drugs (NSAIDs) associate with zwitterionic phospholipids: insight into the mechanism and reversal of NSAID-induced gastrointestinal injury. Nature Medicine 1(2): 154-158.

14. Golub Lorne M, Ramamurthy NS, Thomas F Mc Namara (1991) Tetracyclines inhibit connective tissue breakdown: New therapeutic implications for an old family of drugs. Critical Reviews in Oral Biology \& Medicine 2(3): 297-322.
15. Golub LM, Lee HM, Ryan M, Giannobile W, Payne J, et al. (1998) Tetracyclines inhibit connective tissue breakdown by multiple nonantimicrobial mechanisms. Adv Dent Res 12(2): 12-26.

16. Ryan Maria E, Adil Usman, Ramamurthy NS, Lorne M Golub, Robert A Greenwald (2001) Excessive matrix metalloproteinase activity in diabetes: inhibition by tetracycline analogues with zinc reactivity. Current medicinal chemistry 8(3): 305-316.

17. Golub LM, Wolff M, Lee HM, Mc Namara, Ramamurthy NS, et al. (1985) Further evidence that tetracyclines inhibit collagenase activity in human crevicular fluid and from other mammalian sources. Journal of periodontal research 20(1): 12-23.

18. Golub LM, Ramamurthy NS, Gomes B, Wolff M, Casino A, et al. (1984) Tetracyclines inhibit tissue collagenase activity. J Periodont Res 19(6): 651-655.

19. Golub Lorne M, Thomas F Mc Namara, Maria E Ryan, Bruce Kohut, Timothy Blieden, et al. (2001) Adjunctive treatment with subantimicrobial doses of doxycycline: effects on gingival fluid collagenase activity and attachment loss in adult periodontitis. Journal of clinical periodontology 28(2): 146-156.

20. Gu Ying, Clay Walker, Maria E Ryan, Jeffrey B Payne, Lorne M Golub (2012) Non-antibacterial tetracycline formulations: Clinical applications in dentistry and medicine. Journal of Oral Microbiology 4:1-14.

21. Golub Lorne M, Muna S Elburki, Clay Walker, Maria Ryan, Timo Sorsa, et al. (2016) Non-antibacterial tetracycline formulations: host-modulators in the treatment of periodontitis and relevant systemic diseases. International Dental Journal 66(3): 127-135.

22. Hu Jialiang, Philippe E Van Den Steen, Qing Xiang A Sang, Ghislain Opdenakker (2007) Matrix metalloproteinase inhibitors as therapy for inflammatory and vascular diseases. Nature Reviews Drug Discovery 6(6): 480-498.

23. Golub LM, Ramamurthy NS, Leung M, TF Mc Namara (1990) Low-dose doxycycline therapy: Effect on gingival and crevicular fluid collagenase activity in humans. J Periodont Res 25(6): 321-330.

24. Golub Lorne M, Timo Sorsa, His Ming Lee, Sebastian Ciancio, Darius Sorbi, et al. (1995) Doxycycline inhibits neutrophil (PMN)-type matrix metalloproteinases in human adult periodontitis gingiva. Journal of clinical periodontology 22(2): 100-109.

25. Walker S, Golub LM (2012) Host modulation therapy for periodontal disease: subantimicrobial-dose doxycycline, medical as well as dental benefits. Oral Health Journal 10: 24-35.

26. Golub LM, TF Mc Namara, Angelo GD, Greenwald RA, Ramamurthy NS (1987) A non-antibacterial chemically - modified tetracycline inhibits mammalian collagenase activity. J Dent Res 66(8): 1310-1314.

27. Ryan M (2002) Host modulation: Conceptualization to clinical trials and integration into clinical practice. CDA Journal 30(4): 285-293.

28. Roy Shreyas K, Daniel Kendrick, Benjamin D Sadowitz, Louis Gatto, Kathleen Snyder, et al. (2011) Jack of all trades: Pleiotropy and the application of chemically modified tetracycline- 3 in sepsis and the acute respiratory distress syndrome (ARDS). Pharmacological Research 64: 580-589.

29. Lokeshwar Bal L, Eva Escatel, Baoqian Zhu (2001) Cytotoxic activity and inhibition of tumor cell invasion by derivatives of a chemically modified tetracycline CMT-3 (COL-3). Current medicinal chemistry 8(3): 271-279.

30. Ramamurthy, Nungavaram S, Jing wen Xu, John Bird, Andrew Baxter, et al. (2002) Inhibition of alveolar bone loss by matrix metalloproteinase inhibitors in expeimental peiodontal disease. Journal of periodontal research 37(1): 1-7.

31. Gu Ying, His Ming Lee, Sanford R Simon, Lorne M Golub (2011) Chemically modified tetracycline-3 (CMT-3): A novel inhibitor of the serine proteinase, elastase. Pharmacological Research 64(6): 595-601.

32. Gu Ying, His Ming Lee, Timo Sorsa, Aino Salminen, Maria E Ryan, et al. (2011) Non-antibacterial tetracyclines modulate mediators of 
periodontitis and atherosclerotic cardiovascular disease: A mechanistic link between local and systemic inflammation. Pharmacological Research 64(6): 573-579.

33. Dezube BJ, SE Krown, JY Lee, KS Bauer, DM Aboulafia (2006) Randomized phase II trial of matrix metalloproteinase inhibitor COL-3 in AIDS-related Kaposi's Sarcoma: An AIDS malignancy consortium study. Journal of Clinical Oncology 24(9): 1389-1394.

34. Drucker A, Rosen C (2011) Drug-induced photosensitivity. Culprit drugs, management and prevention. Drug Saf 34(10): 821-837.

35. Richards Christopher, Liron Pantanowitz, Bruce J Dezube (2011) Antimicrobial and non-antimicrobial tetracyclines in human cancer trials. Pharmacological Research 63(2): 151-156.

36. Ryan ME, HM Lee, T Sorsa, LM Golub (2008) Effects of short-term COL-3 on local biomarkers of periodontitis. JDR 87: 0040.

37. Tenenbaum H, A Shelemay B Girard, R Zohar, Fritz PC (2002) Bisphosphonates and periodontics: Potential applications for regulation of bone mass in the periodontium and other therapeutic/diagnostic uses. J Periodontol 73(7): 813-822.

38. Shinoda Hisashi, Sadaaki Takeyama, Keiko Suzuki, Shinobu Murakami, Shoji Yamada (2008) Pharmacological Topics of Bone Metabolism: A Novel Bisphosphonate for the Treatment of Periodontitis. Journal of Pharmacological Sciences 106(4): 555-558.

39. Marx Robert E, Yoh Sawatari, Michel Fortin, Vishtasb Broumand (2005) Bisphosphonate-Induced Exposed Bone (Osteonecrosis/Osteopetrosis) of the Jaws: Risk Factors, Recognition, Prevention, and Treatment. Journal of Oral and Maxillofacial Surgery 63(11): 1567-1575.

40. Woo, Sook Bin, John W Hellstein, John R Kalmar (2006) Systematic review: bisphosphonates and osteonecrosis of the jaws. Annals of internal medicine 144: 753-761.

\section{ISSN: 2574-1241}

DOI: 10.26717/BJSTR.2018.07.001513

Muna S Elburki. Biomed J Sci \& Tech Res

This work is licensed under Creative

Commons Attribution 4.0 License

Submission Link: https://biomedres.us/submit-manuscript.php
41. Serhan Charles N, Nan Chiang, Thomas E Van Dyke (2008) Resolving inflammation: dual anti-inflammatory and pro-resolution lipid mediators. Nature Reviews Immunology 8(5): 349-361.

42. Zhang Yu, Ying Gu, His Ming Lee, Elena Hambardjieva, Kveta Vrankova, et al. (2012) Design, synthesis and biological activity of new polyenolic inhibitors of matrix metalloproteinases: a focus on chemically-modified curcumins. Current medicinal chemistry 19(25): 4348-4358.

43. Anand Preetha, Ajaikumar B Kunnumakkara, Robert A Newman, Bharat B Aggarwal (2007) Bioavailability of curcumin: Problems and promises. Molecular Pharmaceutics 4(6): 807-818.

44. Elburki MS, Moore DD, Terezakis NG, Zhang Y, Lee HM, et al. (2017) A novel chemically modified curcumin reduces inflammation-mediated connective tissue breakdown in a rat model of diabetes: periodontal and systemic effects. Journal of Periodontal Research 52(2): 186-200.

45. Elburki Muna S, Carlos Rossa Jr, Morgana R Guimaraes, Mark Goodenough, His Ming Lee, et al. (2014) A novel chemically modified curcumin reduces severity of experimental periodontal disease in rats: Initial observations. Mediators of Inflammation 2014: 1-10.

46. Elburki Muna S, Carlos Rossa, Morgana R Guimarães Stabili, His Ming Lee, Fabiana A Curylofo Zotti, et al. (2017) A Chemically Modified Curcumin (CMC 2.24) Inhibits Nuclear Factor $\kappa B$ Activation and Inflammatory Bone Loss in Murine Models of LPS-Induced Experimental Periodontitis and Diabetes-Associated Natural Periodontitis. Inflammation 40(4): 1436-1449.

47. Kirkwood Keith L, Joni A Cirelli, Jill E Rogers, William V Giannobile (2007) Novel host response therapeutic approaches to treat periodontal diseases. Periodontology 43: 294-315.

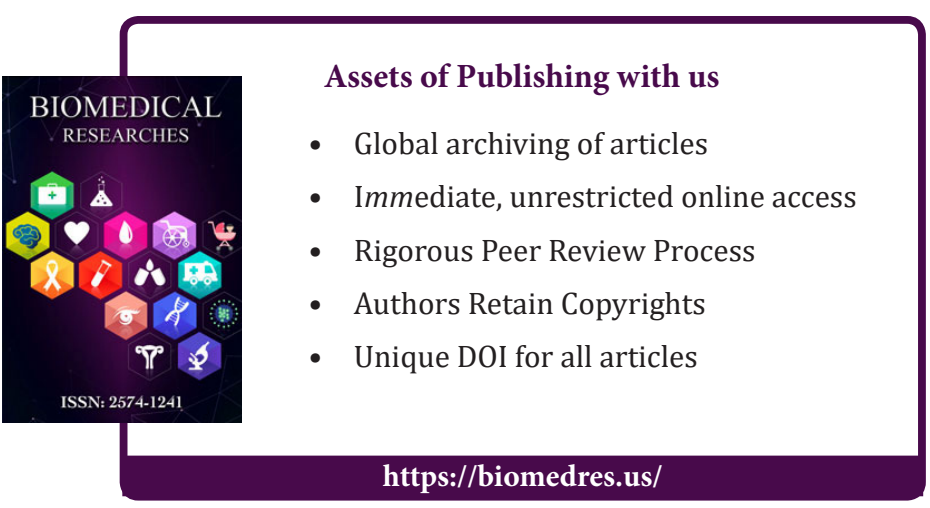

